\title{
دراسة الآثار الاقتصادية للفجوة الغذائية لأهم المحاصيل فى مصر
}

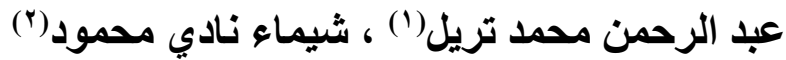

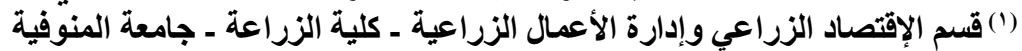

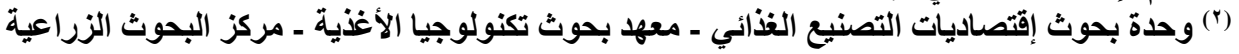

Received: May 27, 2018

Accepted: Jun. 4, 2018

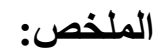

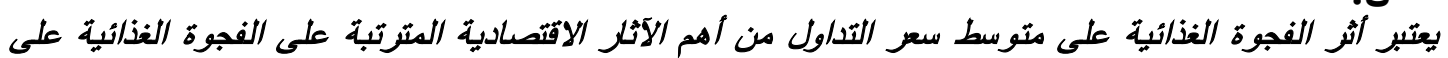

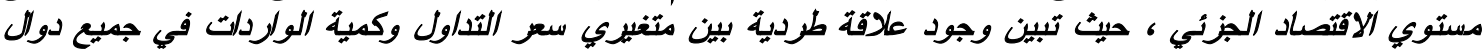

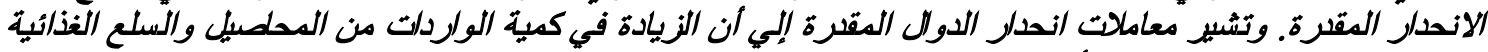

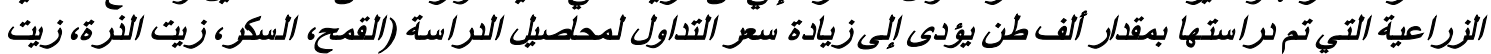

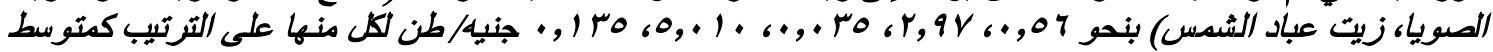

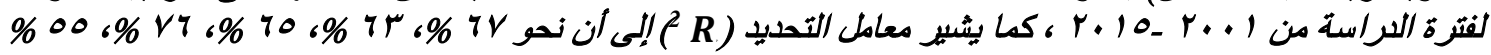

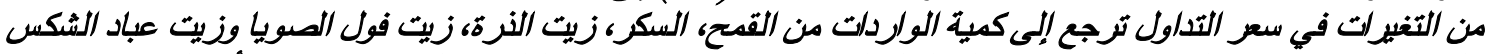

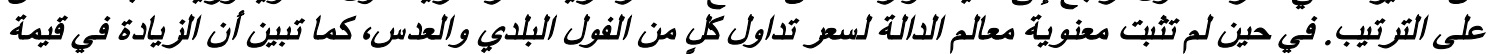

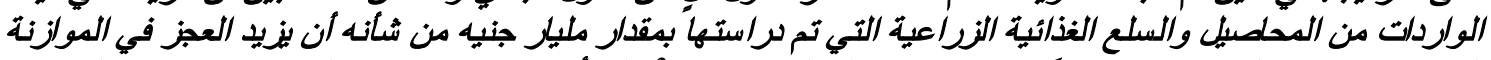

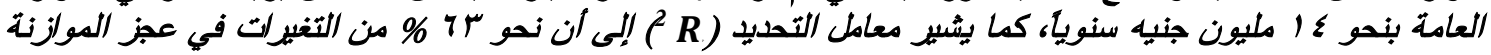

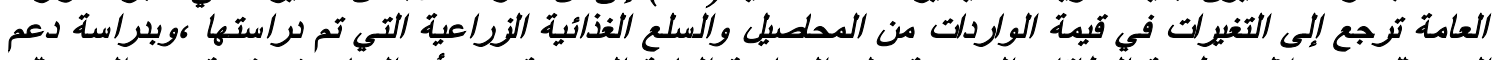

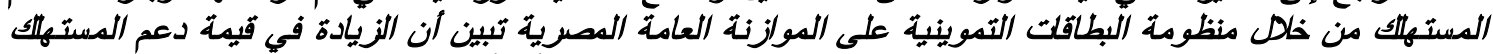

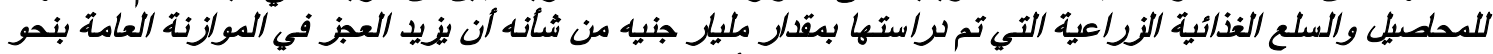

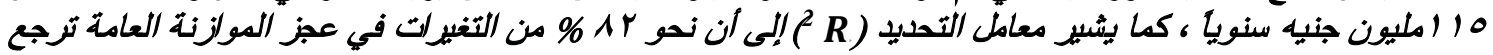

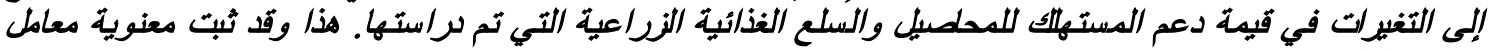

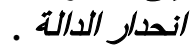

الكلمت الاسترثادية: (المحلصيل الاستراتيجية ـ الفجوة الغذاثية_عز الموازنة العامة ـ الاثر الافتصادية للفجوة

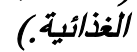

تتمثل مشكلة البحث فى وجود عجز كلى فى الموازنة

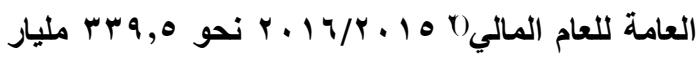
جنيه يعادل نحو r, r \% من الناتج المحلى الإجمالي المقدر للعام ذاته. وكان العجز قد بلغ في العام المالي

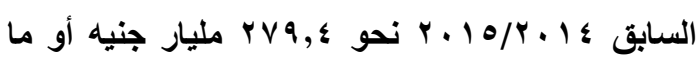
يعادل 0,11\% من الناتج المحلى. ويعتبر العجز في الميزان التجاري المصري أحد الأسباب الهامة للعجز

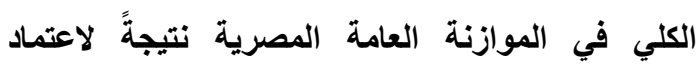
الاقتصاد المصري علي الواردات للد الفجوة الغذائية

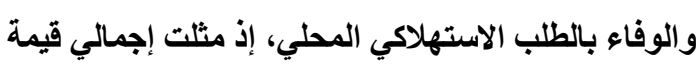

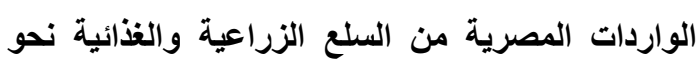

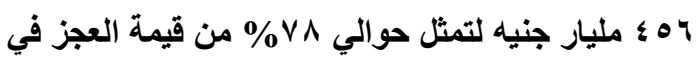

() وزارة المالية "التقرير المالي الثهري "ديسمبر

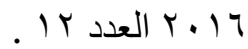

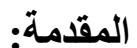

تعتبر الفجوة الغذائية أحد الإثكاليات الهامة التي

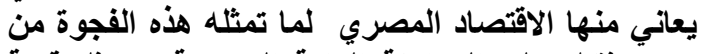

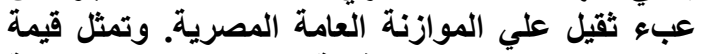
واردات مصر من العلع الغذائية والمحاصيل الاستراتيجية والتئية

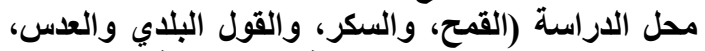

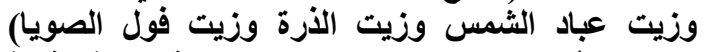

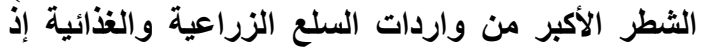

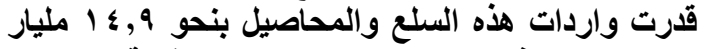

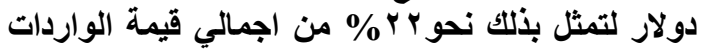

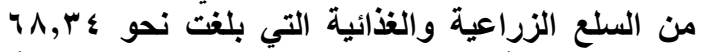

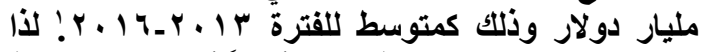

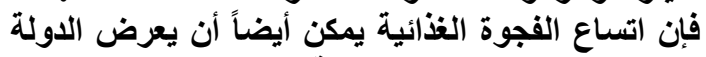

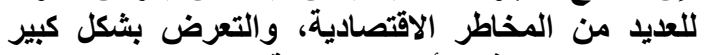
لتقلبات الاسعار في الأسواق الدولية. المشكلة البحثية: 
هذه السلع والتحول نحو بدائل أخري. ويؤدي هذا الأمر

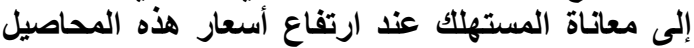

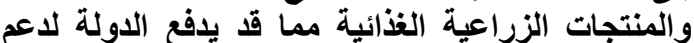

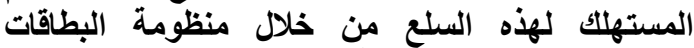

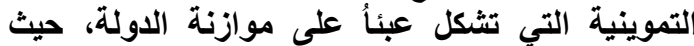

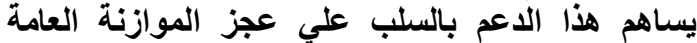

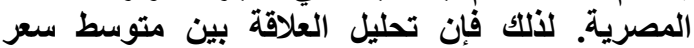
التداول لكل محصول او سلعة غذائية زراعية الثئة الشتملته

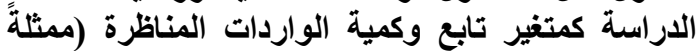

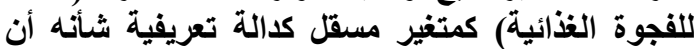
يصف هذه العلاقة، ويوضح مدير ملثي تأثير الفجوة الغذائية

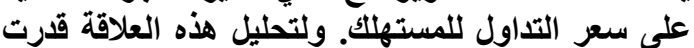

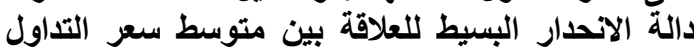
(كمتغير تابع) وكمية الواردات (كمتغير مستقلة) لكل لكئل

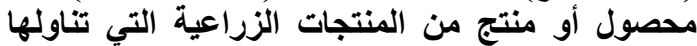

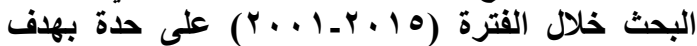

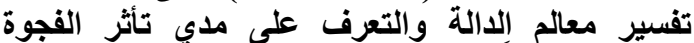
الغذائية متمثلةً في كمية الواردات على سلى سعر تداول

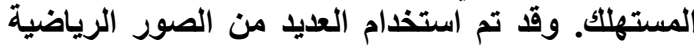

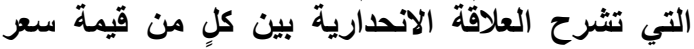

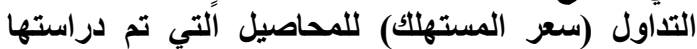

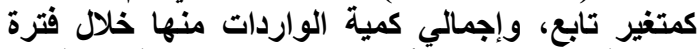

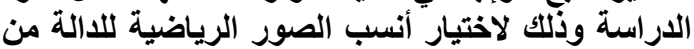

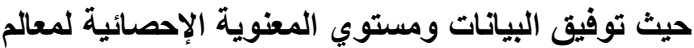

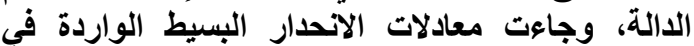

الجدول (1) أنسب الصور الرياضية للمعادلات المقدرة.

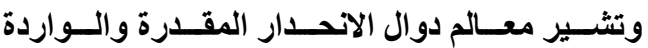

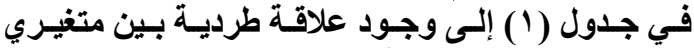

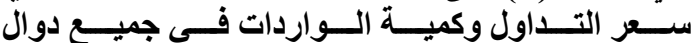

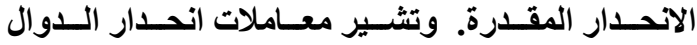

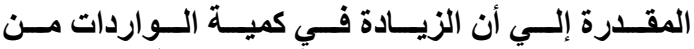

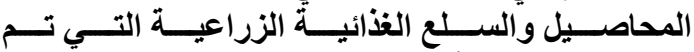

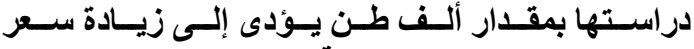

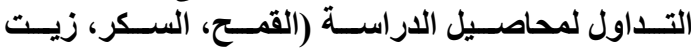

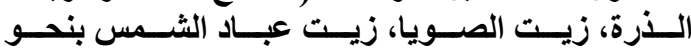

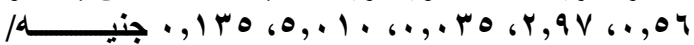
طن كمتوسط لفترة الدراسة على الترتيب.

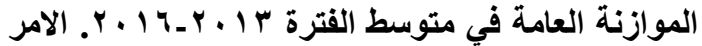
الذي يعرض الدولة للعديد من المخاطر الاقتصادية، ومخاطر تقلبات اسعار السلع والمحاصيل محل الدراسة

$$
\text { في الأسواق الدولية. }
$$
الهدف البحثى:

يستهلف البحث التعرف على الآثار الاقتصادية السلبية للفجوة اللذائية على المستويين الكلي الكئي والجزئئي

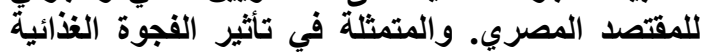

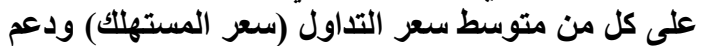

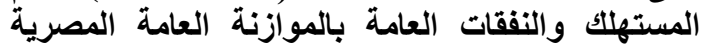

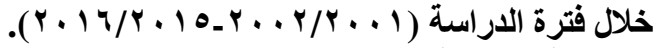
الطريقة البحثية:

تم استخدام العديد من الأساليب الإحصائية الوصفية

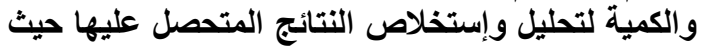

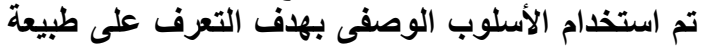

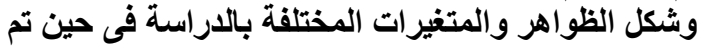

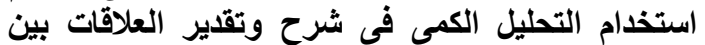
متغيرات النماذج الرياضية المستخدمة ذاتفات الصلاتلة بأهداف الاراسة. مصادر البيانات: اعتمد البحث على البيانات الثانوية المنشورة وغير

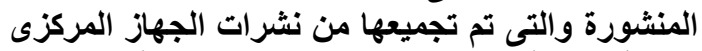

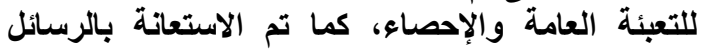
العلمية والأبحاث ذات الصلة بموضع البحث.

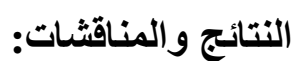

أولاً: أثر الفجوة الغذائية على متوسط سعر

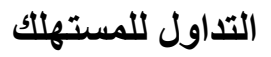

يعتبر أثر الفجوة الفذائية على متوسط سعر التذاول

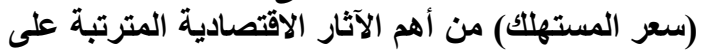

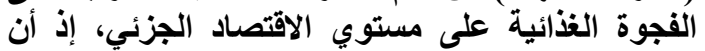

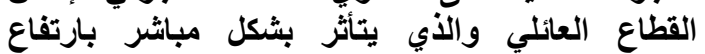

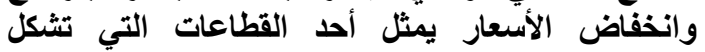

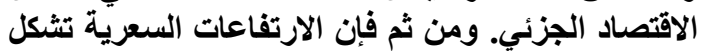
أثراً سلبياً على المستهلك لعدم قدرته على الاستغناء عن تلعن

\begin{tabular}{|c|c|c|c|}
\hline F قيمة F & معامل التحديد R & 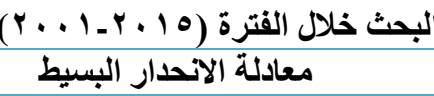 & البيان \\
\hline 24,3 & 0,67 & $\begin{aligned} \hat{Y}_{i}=- & 878.8+0.56 X_{i} \\
& (-1.174) *(4.93) * *\end{aligned}$ & القمح \\
\hline 9,21 & 0,63 & $\begin{array}{c}\hat{Y}=1505.7+2.97 X_{I} \\
(1.94) * \quad(3.03) *\end{array}$ & السكر \\
\hline
\end{tabular}

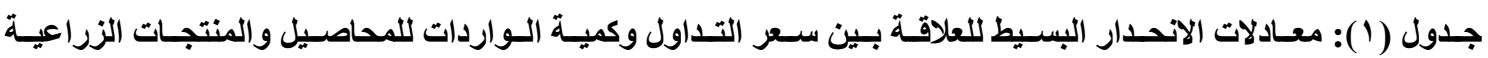




\begin{tabular}{|c|c|c|c|}
\hline 23,6 & 0,65 & $\begin{array}{c}\hat{Y}_{i}=-167.8+0.035 X_{i} \\
(-4.23) * \quad(3.65) *\end{array}$ & زيت الذرة \\
\hline 2,6 & 0,76 & $\begin{aligned} \hat{Y}_{i}= & 63.28+5.010 X_{i} \\
& (0.515) *(1.031) *\end{aligned}$ & زيت الصويا \\
\hline 13,12 & 0,55 & $\begin{aligned} \hat{Y}_{i}= & -102.46+0.135 X_{I} \\
& (-1.059) * \quad(3.62) * *\end{aligned}$ & زيت عباد \\
\hline
\end{tabular}

المصدر: جمعت وحسبت من بيانات الجدول (أ) بالملحق.

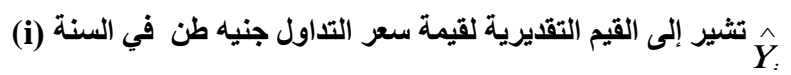
تثبير إلى متغير كمية الواردات خلال فترة الاراسة. ( ألف طن)

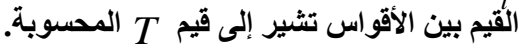
10،...................

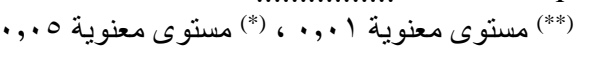

كمتغير مستقل يمكن ان يمدنا بمعالم دالية تفسر العلاقة بين هذين المتغيرين للتعرف علي مدي تأثير الفجوة

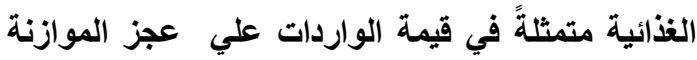
العامة. ويتضح من نتائج تحليل دالة الانحدار البسيط للعجز الكلي كمتغير تابع وقيمة الواردات من السلع الغذائية الاستراتيجية موضع الاراسة كمتغير مستقل

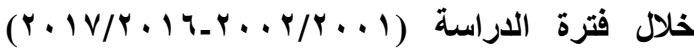
علي النحو الوارد بييانات الجدول رقم (r). ولتقلير دالة الانحدار البسيط بين كل من قيمة العجز الكلي للموازنة العامة المصرية كمتغير تابع، وإجمالي قيمة الواردات من المحاصيل والسلع الغذائية الزراعية التي تم دراستها خلال فترة الدراسة كمتغير مستقل للبيانات الواردة بالجدول رقم (ץ) تم استخدام العديد من الصور الرياضية التي تثرح العلاقة الانحارية، وذلك لاختيار أنسب الصور الرياضية للالة من حيث توفيق البيانات ومستوي المعنوية الاحصائية لمعالم الدالة. وقد اتضح أن الصورة الخطية لهذه العلاقة هي أفضل الصور الرياضية من حيث توفيق بيانات تلك الفترة، ومستوي المعنوي الاحصائية لمعالم الدالة، حيث تأكد ثبوت معنوية

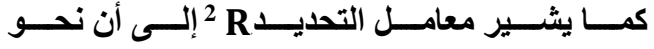

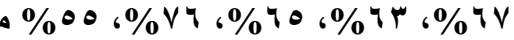
التغيرات في سـعر التـاول ترجيع إلـى كميـة الـواردات

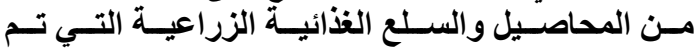
دراستها على الترتيب. في حين لم تثبت معنوية معالم الدالة لكلٍ من سعر التبل

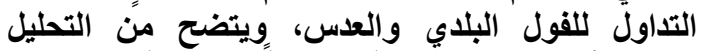

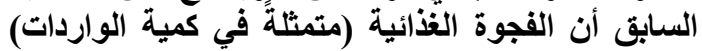
تؤثر بصور مباشرة على سعر التداول (سعر المستهليكتة للمحاصيل والسلع الغذائية الزراعية التي تم دراستها.

ثانياً: أثر الفجوة الغذائية على الموازنة العامة للاولة: الفجرة

يعتبر الإنفاق علي الواردات بصفة عامة أحد بنود الإنفاق في الموازنة العامة .وحيث أن قيمة واردات مصر من السلع الغذائية الاستراتيجية موضع الاراسة ( القمح، والسكر، والقول البلاي، والعدس، وزيت عباد الثمس، وزيت الذرة، وزيت فول الصويا) تمثل الثطر الأكبر من

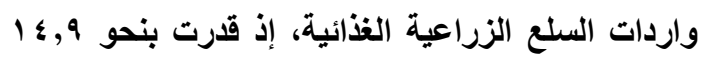
مليار دولار تمثل نسبتها نحو بr\% من قيمة الواردات

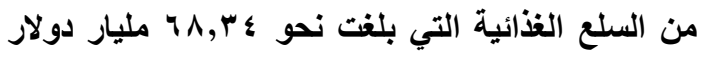

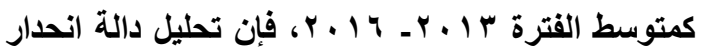
العجز الكلي في الموازنة العامة كمتغير تابع وقيمة الواردات من السلع الغذائية الاستراتيجية موضع الدراسة 


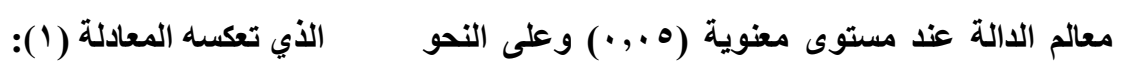

$$
\begin{gathered}
\hat{Y}=1.170+0.014 X 1 \ldots \ldots \ldots \\
(2.58 * \quad(4.72) * * \\
\mathrm{R}^{2}=0.63 \quad \mathrm{~F}=22.23
\end{gathered}
$$

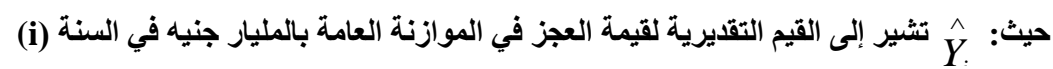
تثثير إلى متغير قيمة واردات محاصيل الدراسة بالمليار جنيه خلال فترة الدراسة.

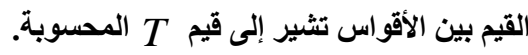

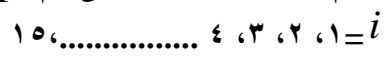

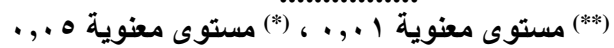

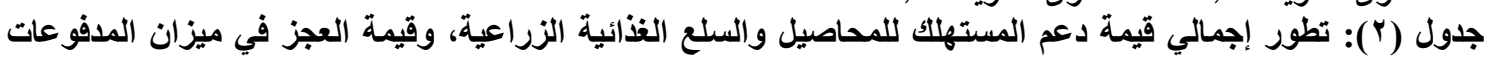
بالمليار جنيه ميزان المدان

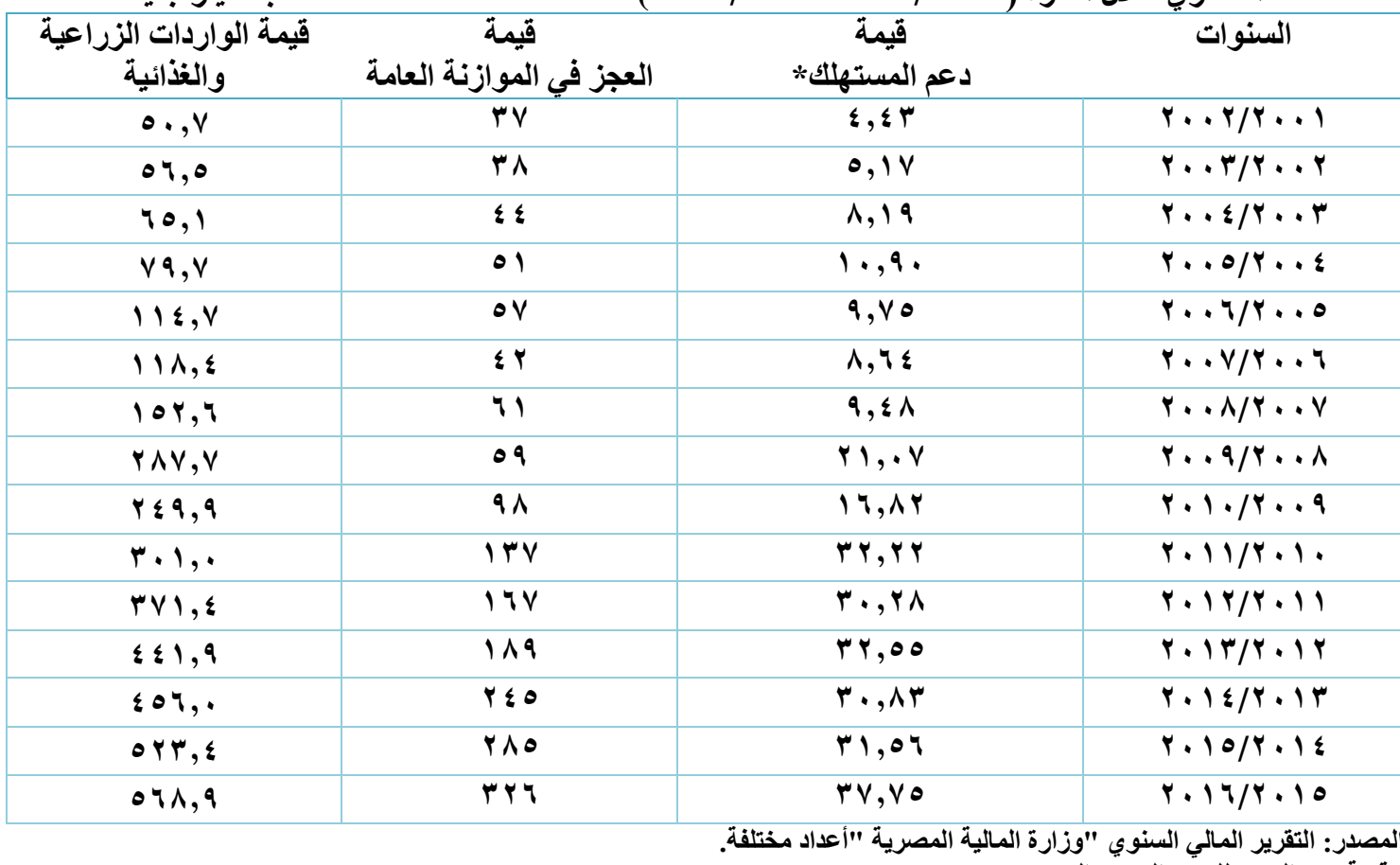
*قيمة دعم المستهلك من الزيت والسنوي والسكر

الآثار الاقتصادية للفجوة الغذائية على مستوي الاقتصاد

الكلي.

ثالثاً: أثر دعم المستهلك من خلال منظومة

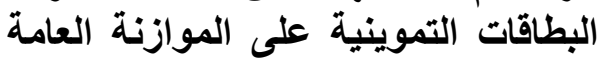

$$
\text { المصرية. }
$$

يعتبر دعم المستهلك من خلال منظومة البطاقات التموينية أحد الأليات التي تستخدم من جاتب الحكومة لتخفيف جاتب من أعباء المعيشة عن كاهل محدودي الاخل ومما لا شك فيه أن هذا الدعم يمثل عبئًاً على اعلى

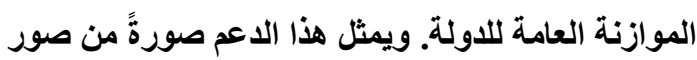

ويتضح من معالم الدالة المقدرة وجود علاقة طردية

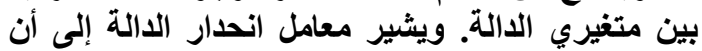

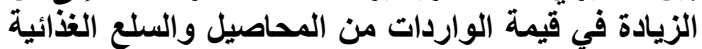

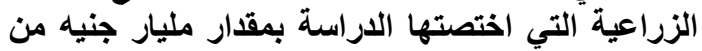

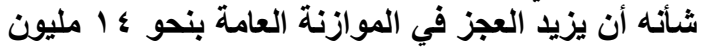

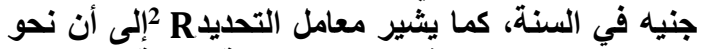

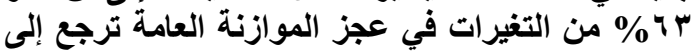

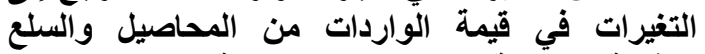
الغذائية الزرأعية التي اختصنها التهات الدراسة.

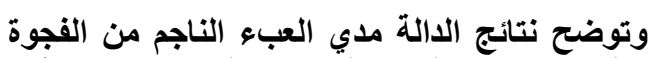

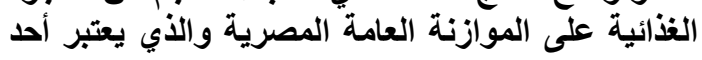


الجدول (ץ) تطور إجمالي قيمة دعم المستهلك للمحاصيل

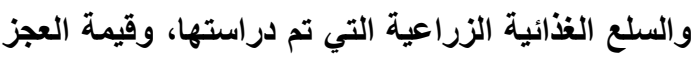
في ميزان المدفوعات المصري خلال الفترة

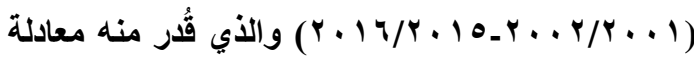
الاتحدار البسيط السابق الاشارة اليها.

وتوضح المعادلة (ץ) معالم الدالة الانحدارية للعلاقة بين قيمة العجز في الموازنة العامة كمتفير تابع، وقيمة العابحة دعم المستهلك للمحاصيل والسلع الغذائية الزراعية التئي التئية اختصتها الدراسة كمتغير مستقل خلال فترة الاراسة.

$$
\begin{aligned}
& \hat{Y}=5.403+0.115 X 1 \\
& \text { (2.39) * (7.710)** } \\
& \mathrm{R}^{2}=0.82 \quad \mathrm{~F}=59.4
\end{aligned}
$$

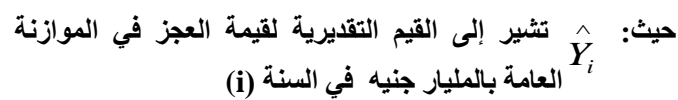

$$
\begin{aligned}
& \text { تثير إلى متغير قيمة الاعم المستهلك من الزيت والسئ والسكر } \\
& \text { بالمليار جنيه خلال فترة الاراسة. }
\end{aligned}
$$

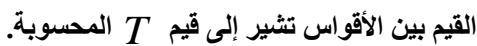

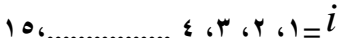

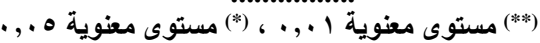

ويتضح من معالم الدالة المقدرة وجود علاقة طردية بين متغيري الدالة. ويشير معامل الدئل الحدار الدالة إلى ألى أن

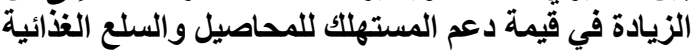

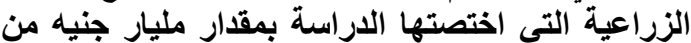

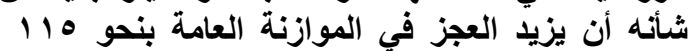

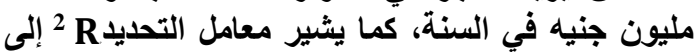

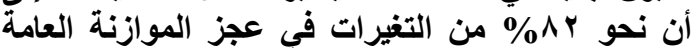
ترجع إلى التغيرات في قيمة دعم المستهليك للمحاصيل

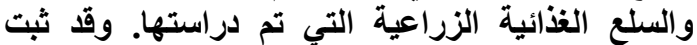

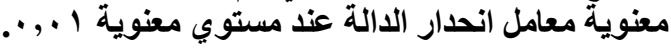

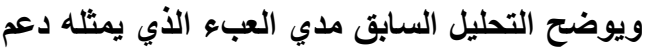

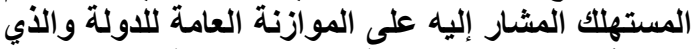
يعتبر أحدالآثار الاقتصادية للفجوة الغذائية على مستوي لألئي الاقتصاد الكلي.

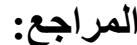
1ـ الجهاز المركزي للتعبئة العامة والاحصاء ـ الكتاب

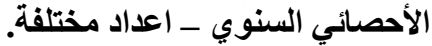
r- وزارة المالية "التقرير المالي الثهري "ديسمبر
الدعم المباثر والذي يتمثل في المبالغ التي تخصصها

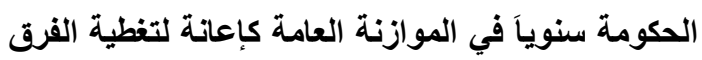
بين سعر السلعة المتداول وبين تكلفتها الحقيقة. وقد بلغت

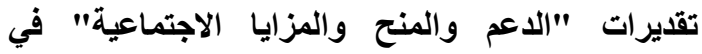

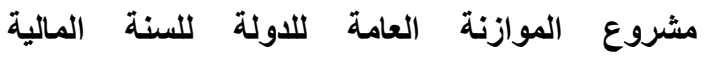

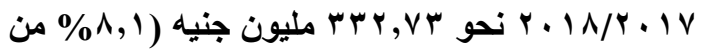
الناتج المحلى الإجمالي)، مقابل نحو \&, צ ـ ب مليون جنيه

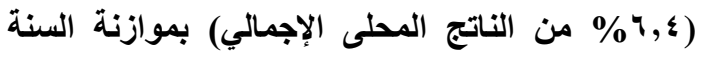

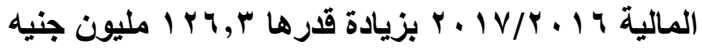
تمثل نحو ץ,آ\% من العام المالي السابق. وتوزع

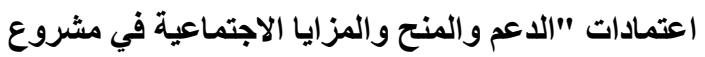

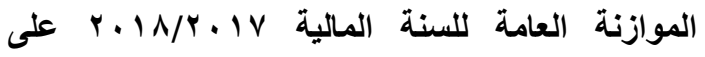
"الاعم السلعي (دعم القمح المستورد، دعم الخبز، دعم الثمانه السكر والزيت التمويني، دعم السلع الاضافية التموينية و هي السكر الاضافي والزيت الحر والأرز والثاي) والذي الذي

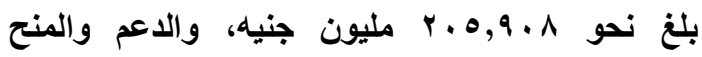

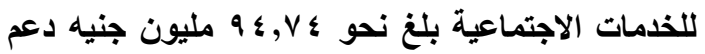

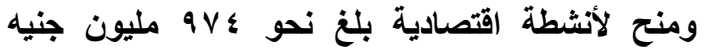

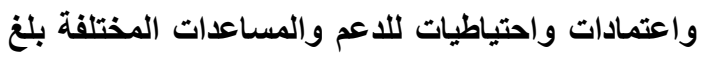
نحو I, Y مليون جنيه". وقد بلغ دعم السلع من خلال

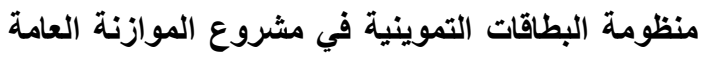

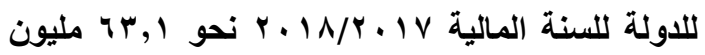
جنيه مقابل نحو rا, اء مليون جنيه بموازنة السنة

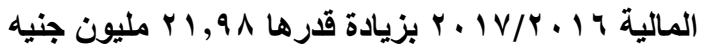
تمثل نحو ه, به\% من نظيرتها في العام المالي السابق. وللتعرف على مدي تأثير قيمة دعم السلع الغذائية على الموازنة العامة تم تقدير دالة الانحدار البسيط بين قيمة العجز في الموازنة العامة وقيمة دعم السلع الغذائية

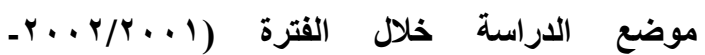
(r. T T/Y. 10 وقد تم استخدام العديد من الصور الرياضية التي تثرح العلاقة الانحارية بين المتغيرين وذلك لاختيار أنسب الصور الرياضية للالة من حيث توفيق البيانات

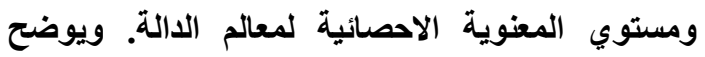




\title{
THE ECONOMICS EFFECTS OF THE FOOD GAB FOR THE MOST IMPORTANT CROPS IN EGYPT
}

\author{
A. M. Taryal and Shiamaa N. M. Mohamed \\ (1) Agri-Economics department -Faculty of Agriculture-Minufiya University \\ (2) Food Technology Research Institute - Agricultural Research Center
}

\begin{abstract}
The impact of the food gap on the average consumer price is one of the most important economic impacts of the food gap at the microeconomic level. There was also a positive relationship between the consumer price and the quantity of imports for wheat, sugar, corn oil, soybean oil, and sunflower oil.

The Regression coefficients indicate that the increase in the quantity of imports of wheat, sugar, corn oil, soybean oil and sunflower oil by 1000 tons leads to an increase in the consumer price by $0.56,2.97,0.035,5.010,0.135 \mathrm{LE} /$ ton respectively .The coefficient of determination $\left(R^{2}\right)$ indicates that about $67 \%, 63 \%, 65 \%, 76 \%, 55 \%$ of the changes in the consumer price of wheat, sugar, corn oil, soybean oil and sunflower oil respectively are due to the amount of imports.

The regression coefficients between the consumer price and the quantity of imports for both beans and lentils are insignificant. This is because the quantity of imports of these
\end{abstract}


crops does not affect the consumer price since these crops are characterized by weak demand elasticity and the inability of the consumer to dispense with them.

The increase in the value of imports (wheat, beans, lentils, sugar, corn oil, soybean oil and sunflower oil) by one billion pounds would increase the budget deficit by about 14 million pounds annually .The coefficient of determination $\left(R^{2}\right)$ indicates that about $63 \%$ of changes in the budget deficit are due to changes in the value of imports.

To study consumer subsidies through the ration card system on the Egyptian public budget shows that the increase in the value of consumer subsidies for crops and agricultural food commodities, which was allocated by the study by one billion pounds, would increase the budget deficit by about 115 million pounds annually. The coefficient of identification $\left(R^{2}\right)$ indicates that about $82 \%$ of changes in the budget deficit are due to changes in the value of consumer support for crops and agricultural food commodities.

Key words: Food gab, the economics, effects of the food gab, the most important, the genral budget deficit

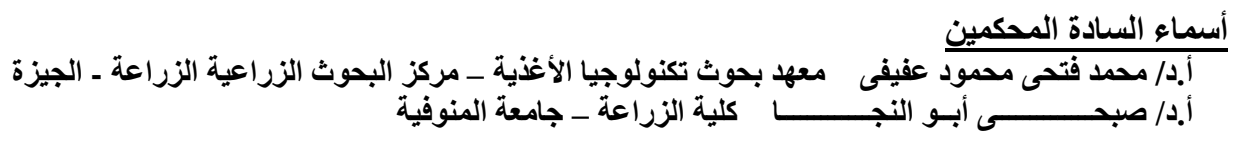


Menoufia J. Agric. Economic \& Social Sci. Vol. 3 August (2018): 4 rl - $\varepsilon$ r8 
جدول ( أ ): يوضح تطور سعر التداول المحلى جنبه/طن وكمية الواردات ألف /طن لكل من (القمح-الفول البلاى ـالعس السكر- زيت الذره - زيت فول الصويا- زيت عباد الثمس) خلال

\begin{tabular}{|c|c|c|c|c|c|c|c|c|c|c|c|c|c|c|}
\hline \multicolumn{2}{|c|}{ زيت عباد الثمس } & \multicolumn{2}{|c|}{ زيت الصويا } & \multicolumn{2}{|c|}{ زيت الذره } & \multicolumn{2}{|c|}{ السكر } & \multicolumn{2}{|c|}{ العدس } & \multicolumn{2}{|c|}{ الفول البلدي } & \multicolumn{2}{|c|}{ القمح } & \multirow[b]{2}{*}{ السنوات } \\
\hline (جنيه/طن) & 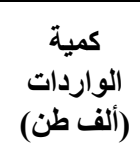 & سعن التداول) & (ألف طنات & |سعر التداول| & (ألف طن) & |سعر التداول) ||(جن/طن) & (النف طن) & |سعر التداول) & (ألف طن) & 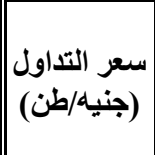 & (الف طمنية & |جنيه/طن) |لتداول) & (الف طمية & \\
\hline Trtr & 09 & rצד & 11. & rorv & 11 & IrY. & ؛ & $r \varepsilon . q$ & $11 \%$ & IAT4 & 198 & $V \Psi \varepsilon$ & YAIA & r... \\
\hline rort & rq & $r q 0 \leq$ & 104 & rarv & r. & Irs. & $0 . \mathrm{V}$ & rooq & $1 \ldots$ & $1 \wedge 1$. & $r \wedge \wedge$ & va9 & sor & $r \ldots r$ \\
\hline$\varepsilon v \cdot \varepsilon$ & IrT & $r .9 r$ & $1 . V$ & ov4. & ir & 19r. & $r{ }_{1}$ & $r q \leq 0$ & 11 & rrva & $r \cdot \Lambda$ & qAv & 2.70 & $r \ldots r$ \\
\hline אודום & 117 & $\varepsilon 90 \wedge$ & ar & 100. & $1 \varepsilon$ & $r \cdot \Lambda$. & $r \cdot v$ & TATs & 19 & rlor & TI & IYYA & STV & r... \\
\hline orAs & 117 & $0 \ldots$ & 11 & $10 .$. & $\varepsilon r$ & rro. & $0 \wedge \varepsilon$ & ruqq & 1.1 & $r \cdot v 1$ & $r_{\lambda}$. & IYTV & OVVT & r...o \\
\hline OVVT & IrV & 0194 & $\leqslant 0$ & 1011 & 10 & TVY. & rq1 & rqrv & VV & एाभ & \&०१ & Irar & OAr. & $r \ldots r$ \\
\hline$T \leqslant T V$ & 101 & $0 . \leqslant 4$ & 99 & $V \varepsilon \cdot r$ & 17 & 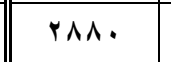 & \&vq & $\varepsilon \cdot r r$ & $\Lambda \varepsilon$ & rrq. & $r \cdot 1$ & vol & 0911 & $r \ldots v$ \\
\hline ATr. & 1199 & גז. & rro & 1109. & $r \varepsilon$ & $r \leqslant \wedge q$ & ITHY & VAqT & $v$. & 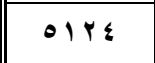 & 900 & $r \cdot \leqslant 1$ & VrAl & $r \ldots \wedge$ \\
\hline $00 .$. & $1 \leqslant 0$ & $00 \ldots$ & 100 & lirr. & YA & rAo. & $\varepsilon .0$ & VAqV & 1.7 & $\{171$ & 011 & $r \leqslant 0 Y$ & Tarr & $r \ldots q$ \\
\hline $0 \ldots$ & 100 & $\Delta 91$. & $19 \varepsilon$ & 1194. & 10 & roro & Av. & VTIE & $1 \times 4$ & STrT & $\leqslant \wedge$. & $19 \leq 4$ & VarA & $r+1$. \\
\hline AAr. & TrT & AAr. & ro. & irva. & $r v$ & \&TVo & Irov & 7979 & qv & OVTs & rir & rors & 9111 & $r .11$ \\
\hline AAT. & $\{\wedge 4$ & 9.9. & $r$ & irar. & o. & \&94 & irva & ITrT & $\Lambda$. & Trar & ro. & TVYS & $90 \leq 9$ & $r .1 r$ \\
\hline 9.9. & ors & $9 \leqslant \wedge$. & IAr & $1 \leqslant 17$. & rq & $\varepsilon 9 \ldots$ & $T \leqslant 1$ & TVIT & 79 & TVq & $\leqslant$ ro & ra१o & $\vee \wedge \vee \wedge$ & $r+1 r$ \\
\hline$q \leqslant \wedge$. & $\leq 4$. & qov. & $1 . r$ & Irk... & rr & OrTV & 914 & ANIO & VV & varl & r.o & קrq. & AIr & $r+1 \varepsilon$ \\
\hline$q 0 \mathrm{~V}$. & $\leqslant 99$ & $1 . \varepsilon \ldots$ & $1 \leqslant r$ & IrAv. & $\mu_{1}$ & OrOA & NIT & 111.1 & $V r$ & noro & rVV & rol. & A.. r & r.10 \\
\hline
\end{tabular}

